\title{
Reducing Hospital Stay in Neonates with Suspected Sepsis
}

\author{
James Somauroo, Linda Nyika - St Peter's Hospital
}

\begin{abstract}
'Suspected sepsis' is one of the most commonly made diagnoses on any neonatal intensive care unit (NICU). Most neonates are discharged after 48 hours following negative blood culture results, thus inefficient analyses of these samples leads to delays in discharge. A baseline study demonstrated that, on average, discharge was delayed by four hours per neonate, with a range of up to 21 hours, with the longest delays being incurred as samples were not being analysed when they were received by the microbiology department out of hours.

Furthermore, problems were due to increase, as the microbiology department was imminently moving to another hospital. In the short term, samples were couriered to another microbiology department, which incubates them overnight. The long-term solution is for the department to purchase their own in-house analyser, for which a business case was developed, presented, and agreed to by the lead neonatal consultant. Unfortunately, the Trust does not believe the venture is financially viable at present.
\end{abstract}

\section{Problem}

'Suspected sepsis' is one of the most commonly made diagnoses on any neonatal intensive care unit (NICU). Treatment of suspected sepsis needs to be timely and effective so that patients may be discharged safely and efficiently, should they proceed to the most common outcome and remain well. Neonates born with suspected sepsis are kept on neonatal units for intravenous antibiotics, pending discharge dependent blood culture results after 48 hours according to Trust policy. However, delays in this process are leading to excess bed days, excess antibiotics being administered, and unnecessary additional stress for mothers and babies.

\section{Background}

In order to understand the process of blood culture analysis, I followed the journey of a sample taken in the hospital. Blood samples were taken immediately after birth, however, as glass was not permitted in the direct, automatic transportation system, or 'pod' system, samples were then placed in a collection tray. Policy stated that porters should pass by this tray to collect any samples and transport them to the laboratory once per hour; however, this timing was unreliable. If the sample was taken during the daytime, it was placed in a queue of jobs in the microbiology laboratory and eventually incubated. A time 48 hours later was then documented and sent to the requesting doctor, via the computerised system as an expected time for culture results to become available. Laboratory technicians worked between $8.30 \mathrm{am}$ and $6.00 \mathrm{pm}$. If the sample was received in the laboratory out of these hours, this process was delayed until the following morning. At this point, technicians were unaware of the discharge dependent nature of the neonatal blood cultures, hence they were not prioritised in the queue of samples that had been collected overnight.

48 hours after blood culture incubation had started, the blood samples were then analysed, and if negative, this data was entered by the technician into the computer system to be seen by the doctor. Following this, all appropriate discharge paperwork was completed and the patient, discharged.

\section{Baseline}

In order to assess the number of patients that this would affect, admissions data was looked at retrospectively for one month. Current hospital policy states that neonates must be kept in hospital for a minimum of 48 hours after birth. On average, four neonates per week were diagnosed with suspected sepsis and discharged with negative blood cultures, with an average stay of 52 hours after birth, four hours more than necessary according to policy. The range of delay was up to 21 hours. In no cases did a neonate have positive blood cultures in this sample; however, in two cases antibiotics were continued on clinical grounds.

\section{Design}

The microbiology department is soon to be moving away from the current hospital, to one 16 miles away, with no current plan as to how this will be managed. It has become apparent during the analysis of this problem that the impending change of microbiology location is going to have a profound effect on delay times, and that both short term and long term solutions are going to be needed to fix a problem that may become worse. Following extensive discussions with pathology, microbiology and neonatal departments, the following solutions emerged:

While microbiology is in the current hospital, and during normal hours, a porter must be called immediately to collect the sample, the microbiology laboratory must also be called to prioritise the sample, and discharge paperwork should be completed in anticipation of discharge if the neonate is clinically well in the hours leading up to potential discharge. 
Out of hours, the sample must be taken to the laboratory before the morning, but the laboratory must be called to prioritise the sample in the meantime. When the microbiology department is moved to its new hospital, in the short term, a courier service, that will also operate overnight, will be run to the new microbiology department with overnight incubation. This is only likely to be a temporary solution to the problem for two reasons; firstly the transit time to the laboratory will be around one to two hours. Secondly, communication between different hospitals and departments will be difficult due to the distance between the units. However, with overnight incubation of cultures, a significant delay problem will be resolved.

\section{Strategy}

The key to this project was developing a long term solution to the foreseen delay problems. The hospital to which the samples will be couriered is 16 miles (approximately 25 minutes) away so delays will occur in transit. Therefore, obtaining a blood culture analyser for the NICU department, housed in the department itself was thought to be the most appropriate solution. This would effectively reduce all delays and mean that if an organism were present in the blood, the organism would be incubated earlier and therefore immediately given the environment to start growing. Neonates would therefore have the greatest chance of being discharged without delay. In order for this to be considered, a business case will be developed with the help of colleagues in the finance department, and will then be presented to the neonatal department.

\section{Post-Measurement}

As mentioned previously, in order to highlight the cost effectiveness of purchasing a blood culture analyser, a business case was created. An outline of which is explained below:

The proposed service will include the leasing of a BD Bactec 9050 blood culture analyser for the processing of urgent NICU blood culture samples. This is a compact analyser that can incubate up to 50 samples.

Based on an average of 3 bed days saved per week, an estimated savings worth $£ 88,140$ ( $£ 565$ per day $x 1$ days saved $x 3$ neonates $x 52$ weeks) per annum can be made if negative blood culture results are reported within 48 hours even if incubated overnight. This also includes an estimated $£ 137.28$ saving on antibiotic costs, offset by a $£ 910$ cost of consumables and rental of the analyser, which is $£ 5580$ per annum on a 3-year contract. By reducing the length of stay per neonate, productivity throughout NICU can be increased, as more neonates can be admitted with suspected sepsis, rather than being sent to other hospitals.

With the maternity unit being expanded, the number of neonates requiring treatment for suspected neonatal sepsis is set to rise, putting even more pressure on the need for bed availability on the neonatal unit. Increasing productivity on NICU will therefore allow the ward to cope with increased demand on services and generate additional income that is above the anticipated £88,140 mark.

\section{Lessons and Limitations}

From start to finish, this project gave me a flavour of how difficult and slow it can be to effect real change, and large numbers of parties involved means exponential numbers of meetings and makes things seemingly impossible at times. It has shown me, however, once change is made, and positive results seen, the reward is much greater.

\section{Conclusion}

The problem that we started with was that there was an unacceptable delay in discharging neonates with suspected sepsis, mainly due to inefficient processes in blood culture transit to the laboratory, and delayed analysis. With services moving to a completely new site, a short term solution to blood culture analysis had to be made, which was to courier samples to the site, and to ensure overnight incubation. This was achieved. A long-term solution of purchasing a blood culture analyser has now been accepted by the department, however, deemed too expensive by the Trust at present.

Blood cultures are now couriered and incubated overnight. There were very few realistic alternatives to this option, so once the microbiology department relocated, this became the practice.

Once the blood culture analyser has been purchased, I suggest that an audit should be completed to analyse the improvement in delay times between couriering to a new hospital 16 miles away and putting them into an on-site analyser. It is clear that there will be a very large saving in time and cost. Interestingly, new NICE guidance has recently been released, which emphasises reducing the necessary inpatient stay time from 48 hours to 36 hours (NICE clinical guideline 149). These should be integrated into the department, and the journey of a blood sample further analysed to find the most efficient method of analysis for discharge.

\section{References}

Polin, R. (2012) Management of Neonates With Suspected or Proven Early-Onset Bacterial Sepsis. Paediatrics, 129:1006

NICE (2012) Antibiotics for early-onset neonatal infection; Antibiotics for the prevention and treatment of early-onset neonatal infection. Nice Clinical Guideline 149. 